\title{
RESEARCH AND DEVELOPMENT OF A STEPPED HEAT PUMP UNIT WITH A HYDRODYNAMIC CAVITATION DEVICE IN SYSTEMS COMPLEX
}

\author{
Anastasia Asmankina \\ Department of information technologies and electronics \\ Volodymyr Dahl East Ukrainian National University \\ 59-a Central ave., Severodonetsk, Ukraine, 93400 \\ Sergiy Korolevsky \\ Department of information technologies and electronics \\ Volodymyr Dahl East Ukrainian National University \\ 59-a Central ave., Severodonetsk, Ukraine, 93400 \\ Marina Loriia \\ Department of information technologies and electronics \\ Volodymyr Dahl East Ukrainian National University \\ 59-a Central ave., Severodonetsk, Ukraine, 93400 \\ Oleksiy Tselishchev \\ Institute of international relations \\ Volodymyr Dahl East Ukrainian National University \\ 59-a Central ave., Severodonetsk, Ukraine, 93400 \\ Andriy Zhydkov \\ Department of Engineering \\ Volodymyr Dahl East Ukrainian National University \\ 59-a Central ave., Severodonetsk, Ukraine, 93400 \\ asmankina@ukr.net
}

\begin{abstract}
Modern European and Scandinavian countries have advanced very far in the development and application of alternative energy sources. The goal of all modern developments is quality improvement and reducing production costs. The precedent of increasing tariffs for utilities is one of the main problems, so there is a need to create non-volatile, autonomous systems that will be controlled and managed remotely. The creation of combined systems that can operate remotely and independently of direct energy resources will lead to a significant increase in the level of protection from the instability of temperature fluctuations and differences in the electrical network.

The present invention relates to heat engineering, in particular to methods and apparatus for generating heat generated differently from combustion of fuel, and can be used in a heating and hot water supply system for residential and industrial premises, as well as for preheating and improving the rheological properties of oil and petroleum products. The presence of sensors in this system allows to monitor, regulate and signal its status, and also allows to set the necessary parameters.
\end{abstract}

Keywords: hydrodynamic unit, refrigerant, thermal coefficient, truncated cones, hydrodynamic cavitation reactor.

\section{Introduction}

The urgency of developing and creating promising sources of power supply for autonomous electricity consumers has long been recognized in many industrialized countries. The limited availability of fossil energy sources, as well as the current situation with rising prices for hydrocarbon fuels along with the exacerbation of the environmental situation, are the factors that stimulate the introduction and use of renewable natural resources in the production of electricity. The main consumers of all types of energy and energy carriers are enterprises, and an indispensable part of any enterprise is its energy economy. It is a collection of generating, converting, transmitting and 
consuming power plants, through which the enterprise supplies all necessary types of energy to the enterprise and uses it in the production process. In addition, the energy sector includes automatic control devices and systems with their information support, non-power plants, buildings, facilities and resources that ensure reliable and economical operation of the company's energy, as well as electric lighting, heating and fuel supply.

The presence of consumers (small towns, villages) located in areas isolated from existing energy networks, or supplied with electricity, for various reasons with interruptions require the organization of autonomous power supply. Let's consider systems in a differentiated form. The first is the heating system of dwelling. The heating and cooling system of a dwelling is exactly that branch of engineering support for buildings, in which the principles of "intelligent building" or "smart house" were first introduced, since this is the main item in the cost of building maintenance. Expensive equipment and installation should be designed for a rather long time to failure. The heating system should itself correct the temperature in the house, reacting to a cooling or thaw. Given the temperature outside the house, the system must regulate the operation of all heating elements so that the room temperature remains as comfortable and optimal as possible. A heating system with built-in intelligence will significantly save money.

Let's consider an example of a heat pump: one liter of water with a temperature of $1{ }^{\circ} \mathrm{C}$ is approximately $4.192 \mathrm{~kJ}$ of heat. The same energy in $4.192 \mathrm{~kJ}$ will have 100 grams of water, but with a temperature of $10{ }^{\circ} \mathrm{C}$. The fact that the heat pump provides hot water with a temperature of $60{ }^{\circ} \mathrm{C}$ does not mean that it simply heated it with electricity by $50^{\circ} \mathrm{C}$. The heat pump spends electricity only on compression and heat transfer, and itself is warm - free. Thus, heat pumps produce $10 \mathrm{~kW}$ of heat, consuming $1.5-2.5 \mathrm{~kW} / \mathrm{h}$ of electricity.

And in practice it looks like this: underground water is below the ground. The temperature of such water is stably high - about $10^{\circ} \mathrm{C}$. After the heat pump does its work (compression), the water temperature at the outlet in the heating circuit will be already $60{ }^{\circ} \mathrm{C}$. It should be noted that the water itself can't be compressed. The mediator (freon) is compressed, which is sensitive to temperature. It will easily take heat from the groundwater through the evaporator (from about $10^{\circ} \mathrm{C}$ through the freon loop it will be selected about $4{ }^{\circ} \mathrm{C}$ ) and also gives the received heat to the heating circuit through the condenser. [1] That is, the principle of the heat pump is the reverse Carnot cycle, and when the heat pump is operating for conditioning - the Carnot cycle itself. In the case of a failure of the heating equipment due to a malfunction or power failure, the system will inform and stop the functioning of the faulty components, but this can be avoided.

The second system is an autonomous power supply system. The problem of energy consumption has been and will be relevant at any time. Adding several autonomous energy sources (for example, solar panels, windmills) and battery to the system increases the stability of the system. The production of photovoltaic cells and solar collectors develops in different directions. Solar batteries come in various sizes: from embedded in micro calculators to roof-topping cars and buildings. The sun is an environmentally friendly source of energy that does not pollute the environment. The operation of solar panels does not lead to greenhouse gas emissions or waste generation, solar energy is inexhaustible, unlike traditional fuels, solar panels after installation require minimal maintenance and produce energy without human participation, among other advantages of solar batteries is worth noting the long life time. It is 25 years or more without degradation of performance. And the use of solar energy is subsidized by the state. For example, in France, for installation of the battery at home, up to $60 \%$ of the cost is reimbursed.

The need to install a windmill near a private house can arise in two cases - if there is no centralized power supply at all or it leaves much to be desired, or you decided to save considerably on paying for electricity. The wind is an ecologically clean, endless source of energy that humanity has enjoyed for thousands of years. Trends in the development of technology unequivocally point to the prospect of using a variety of alternative sources for the full or partial supply of electricity and heat to the house: solar panels, wind generators, thermal convectors and new efficient materials for thermal insulation.

According to this work, a system analysis of existing units is conducted and methods of their improvement for creating a certain set of systems are considered. This unit refers to equipment for 
heating residential and industrial buildings. The compression heat pump comprises an evaporator, a compressor, a condenser, a throttle valve and a liquid separator. The evaporator and the condenser are made in the form of shell-vortex heat exchangers containing supply and discharge nozzles of the working agent and supply and discharge branch pipes, respectively of low potential coolant and high-potential heat carrier, a cochlear-shaped manifold with a guiding apparatus and end walls, and the casing is installed on the outside.

Another unit relates to heat power engineering [2], in particular, to heating installations, hot water supply for small production facilities, individual houses and individual structures using low-potential natural heat sources, domestic wastewater and other heat waste. The method for utilization of low-potential waste water is realized using a heat pump and an external heat exchanger placed inside the hollow column of the airlift submerged in sewage. This makes it possible to utilize the low-potential heat of sewage that has been unclaimed until recently, in enormous quantities continuously sent to the sewage networks of megacities, small towns and workplaces. At the same time, due to this property of the refrigerant used in heat pumps, as its ability to evaporate at a temperature of $3-5^{\circ} \mathrm{C}$, they can utilize the heat of waste water with a temperature not exceeding $5-8{ }^{\circ} \mathrm{C}$ [3].

The heat supply method is considered, which included supplying water for heating to the system, heating water with a single heat pump and delivering the heated water to consumers. To improve the efficiency of the transformation of thermal energy, different methods are used. For example, in the method for achieving the maximum heating coefficient of a heat pump according to the patent [4], the coolant of the heat pump is selected to be liquid so that its critical temperature is close to or equal to the temperature of the cooled medium.

A heat supply method is known which includes supplying water for heating to the heat pump system of the system, heating the water with it and delivering the heated water to consumers. The heat pump system consists of heat pumps, each of which is used as a stage of consecutive water heating. [5] The disadvantage of this method is the organization of the operation of this unit in terms of the heat-transfer cycle, which is close to the quadrilateral Lorentz heat pump cycle. Such cycle, while providing energy savings, but increases the payback period to a value that exceeds the period of economic feasibility, with an increase in capital costs. Thus, the regime of the known heat supply method with such stepwise heating of water does not ensure a positive technical and economic result when it is realized. When using this method of heat supply for heating, the operation of the stepped heat pump system proceeds with large throttle losses of the working fluid in the heat pump circuits, the more the condensation temperature of the working fluid, the greater the throttling losses that can't be eliminated constructively. The disadvantage of this unit is the impulse with the intervals equal to the time of the working cycle, the supply of heat to the heated medium and the inability to use the gas work during its expansion.

The closest is the known heat exchange unit [6] comprising a heat pump including a compressor connected to the compressor heat exchange cavity in which two heat exchangers are arranged: the first heat exchanger is connected to a heat exchanger for performing heat exchange with the external medium and the second heat exchanger is connected to a heat exchanger to perform heat exchange with the consumer. Chlorides are used as a working substance. To move the compressor piston, when compressing the working substance of the heat pump, a hydraulic system is used to supply the working fluid under pressure to the compressor over-piston cavity and the drains of the working fluid from the compressor over-piston cavity. In this heat exchanger, there is no expenditure of energy to push the working medium through the heat exchange circuit of the heat pump, which reduces the energy costs when the heat pump operates.

The aim of this research is development of an efficient source of energy supply, which is a set of systems generating, converting and consuming energy devices, through which the supply of objects with the necessary energy, with further remote control.

\section{Methods}

The main direction of improving heat exchange systems using heat pumps is an increase in the thermal coefficient: the ratio of the amount of heat (cold) transferred to the consumer, to the energy costs for the heat pump operation. 
Therefore, another similar heat exchanger for water heating, containing a closed loop filled with a working substance, is investigated. It includes a compressor for compressing the working medium, a heat exchanger for performing heat exchange with consumers, connected to the compressor outlet, a throttling device at the outlet of this heat exchanger and a heat exchanger-evaporator for performing heat exchange with the external environment [7]. This heat exchange system uses the classic heat pump scheme. A high heating coefficient is achieved by using the swinging in the heat pump of the working substance through a closed circuit, which leads to an increase in the energy consumption for the operation of the heat pump.

Cavitation phenomena, consisting in the formation of local regions in the liquid in which steam-gas caverns are separated and subsequently destroyed, are the result of a fast-flowing process of vapor condensation and collapse of bubbles accompanied by high-frequency hydraulic shocks and high pressure drops in the local area [8].

A hydrodynamic cavitation reactor was studied [9], containing a pipeline in the form of a Venturi pipe, a honeycomb rectifier and cavitators installed in the flow chamber of a Venturi tube. The cavitators are mounted on the axial rod in three rows and are disks or cones serving to turbulize the flow of liquid and the formation of caverns on the rear side thereof. When "collapse" the cavities in their volume, the pressure and temperature increase, which acts on the properties of the treated liquid. Disadvantages of the known device: cavitators have almost no effect on increasing the fluid velocity and, therefore, on reducing the pressure in the liquid, which reduces the intensity of liquid heating and the efficiency of the device will be negligible.

Unit in which, in order to increase the temperature of the liquid heating, an insert is also used, made in the form of a perforated partition installed in the injection pipe. When liquid passes through the channels of the septum, toroidal cavities (cavitation bubbles) are formed in the liquid, pulsating at the outlet of the jets along their periphery. In the caverns, electric discharges occur with a high frequency, the energy of which, when the caverns "collapse", turns into a thermal one, which generates heat in the liquid. The disadvantage is the absence of cavitation in the entire volume.

The device is considered to consist of inlet and outlet nozzles and a cavitator installed in the connecting flanges. In the cavitator, along the concentric circle, channels are formed that expand to the outlet of the liquid. At the entrance to the cavitator there is a reflective cone, which narrows the living section of the inlet branch pipe, entering the inlet, where the liquid increases and the bubbles collapse with the heat release. The disadvantages are: low activity of the cavitation process and a small volume of cavitation zones.

The invention, relating to cavitation generators for dispersing gas (vapor) into a liquid has been investigated. Cavitation bubbles with an energy reserve of up to $100 \mathrm{kcal} / \mathrm{kg}$ under conditions close to normal, move through the liquid to the treated surface. The surfaces initiate the collapse of the vapor bubbles and, by giving their energy higher than the binding energy in the liquid and in the crystal lattice, lead to rupture of the surface of continuity in the liquid. The disadvantage of this device is the lack of localization of the place where cavitation caverns originate.

The closest to the technical solution is the hydrodynamic cavitation reactor, which contains a flow chamber with a cavitator installed inside the inlet and reactive components. [10] The disadvantage is the low efficiency and the cavitation degree of the flow.

\section{Results}

Increasing the efficiency and degree of cavitation of the coolant is achieved by intensifying the process at the interface between phases due to the kinetic energy of the interaction of the fluid flow and the cumulative effect of the collapse of the strings. [11]

Fig. 1 schematically shows a hydrodynamic cavitation reactor.

Reactor contains: a branch pipe of the inlet 1 of the second coolant flow; cover 2; flow chamber 3; cylinder 4; converse truncated cones 5; caverns of collapse 6; nozzle 7; divider 8; separating perforated baffle 9, which serves as a support for the divider; diffuser 10; the outlet pipe of the total flow of the heat carrier 11, consisting of the first and second flows; the inlet pipe of the first heat transfer medium 12 . The circulating heat pump 8 passes the heat transfer medium to the separator 9 , in which the first flow is directed to the branch pipe 12, and the second flow is directed to the branch pipe 11 (Fig. 1). 
Fig. 2 shows a laboratory installation for investigating the input and output parameters of the test system.

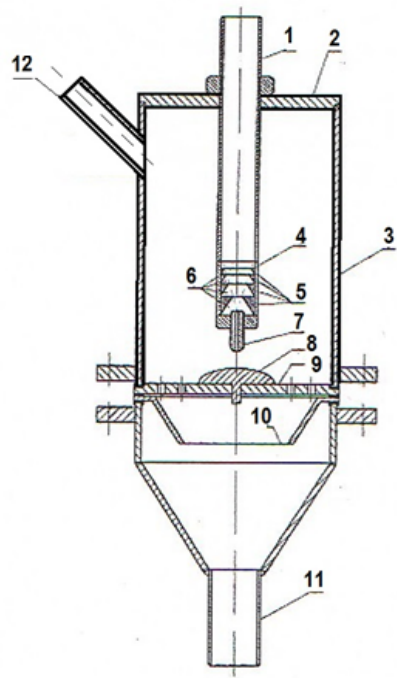

Fig. 1. Hydrodynamic cavitation reactor

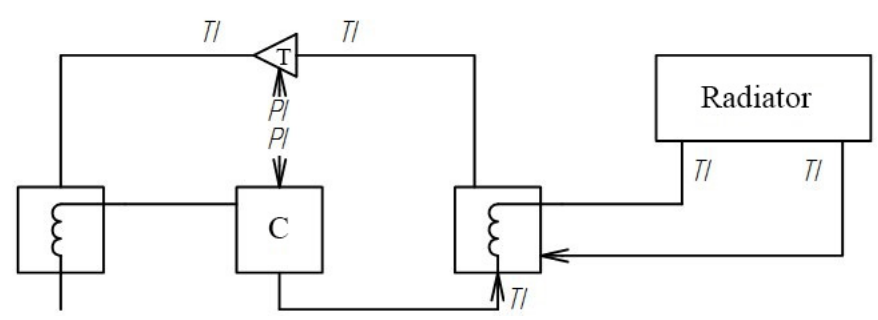

Fig. 2. Test unit of the heating system, where $\mathrm{K}$ - compressor, $\mathrm{T}$ - throttle, TI - temperature sensor system, PI - pressure sensor system

To move the liquid through the pipes a pump is provided at the entrance of the laboratory unit. Water entering the plant passes through the compressor, where compression and movement of refrigerant vapors occurs, as in refrigeration plants. When the vapor is compressed, not only the pressure but also the temperature increases. After the compressor, the compressed refrigerant enters the condenser, where the compressed gas is cooled and converted to liquid, then the liquid flows through the throttling device to the evaporator (where its pressure and temperature decrease), where it boils, passes into a state of gas, thereby taking heat from surrounding space. After this, the refrigerant vapor is returned to the compressor to repeat the cycle. Thus, at the outlet, water will have a temperature much higher than at the inlet, which ensures the heating of the radiator. [12] Then the used and temperature-lowering liquid passes through the throttle to create a hydraulic resistance to the flow of liquid. Additional hydraulic resistance is created by changing the fluid flow cross-section. By changing the hydraulic resistance, a necessary pressure difference is created, which leads to an even greater reduction in the flow temperature.

\section{Discussion}

The developed systems complex allows to heat and cool rooms regardless of the source of the centralized power supply, and the addition of a hydrodynamic cavitation reactor provides the system with greater efficiency.

The hydrodynamic cavitation reactor (Fig. 1) operates as follows. The first flow along the branch pipe 1 is fed to the cylinder 4 , where the reverse truncated cones 5 are installed, which by their horizontal and inclined surfaces form the "caverns" of collapse 6 . The horizontal surfaces of the truncated cones act as a brake element in the form of annular projections, in the course of which 
n cavities (caverns) are formed [13]. The heated stream is forced into "cavities" and, falling into the zones of their closing due to flow turbulence and the presence of a large number of cumulative microstructures formed during the collapse of cavitation bubbles is subjected to the formation of elevated temperatures. In this case, the nozzles formed by the conical surfaces provide the conditions for the appearance of a "secondary" high-speed jet of the nozzle 7.

At some distance from the end surface of the nozzle 7, the hollow conical shell of the liquid jet at a velocity $\mathrm{v}_{0}$ collapses and a secondary high-velocity jet of radius $\mathrm{r}_{0}$ is formed. At the moment of the collapse of the flow of the working medium, the velocity of the secondary high-velocity jet $\mathrm{v}_{\mathrm{c}}$ increases by approximately an order of magnitude with respect to the velocity $\mathrm{v}_{0}$, which follows from the hydrodynamic theory of jets [14].

Further, the secondary high-velocity jet is directed to the divider 8 . Attention was drawn to the place of impact of the jets with a flat surface and the appearance of so-called "wings" - structures resembling the feather of an arrow. It is energetically more expedient to implement a collision with a curved surface and achieve a high level of navigation. In addition, the obtained mixture of the first and second flow coolant passes through the separating baffle 9, passes the diffuser 10, and is discharged through the outlet pipe 11. The efficiency of the heat exchange capacity on the surface of the smallest drops is further enhanced by the Thomson effect - the influence of surface tension forces.

At the moment of the bubble disappearance (at the moment of its collapse), the kinetic energy is transformed into collision energy of elementary particles. The energy released when a bubble collapses is several orders of magnitude higher than the binding energy of elementary particles (nucleons) in the nucleus. As a result of the collision of the nuclei of the claimed method, the energy released between the elementary particles is converted into thermal energy in the liquid and it is withdrawn from the processing zone of the hydrodynamic cavitation reactor.

\section{Conclusions}

The result of this work is development and testing of an experimental laboratory unit. The analysis of the existing developments of autonomous power supply systems and the characteristics of the produced equipment revealed the main trends in the development of this area, to form a promising direction for the practical implementation of the unit itself to take readings from the sensors.

The strong side of this research is the positive effect obtained from reducing the energy load on the compressor. The same decrease in the throttle losses of working heat in the heat pump circuits is achieved at medium condensation temperatures. The increase in the thermal coefficient of the whole unit takes place in a hydrodynamic cavitation reactor, which is considered by the second power plant, which is very important for heating houses, cottages and, in particular, for preheating and improving the rheological properties of oil and petroleum products [15]. The weak side of research is the collection of results for the further development of the mathematical model of the heating installation. To assess the adequacy of a mathematical model, the collection of readings from sensors varies depending on the temperature of the external environment, which requires more detailed observations depending on the time of year and the desired temperature in the room.

\section{References}

[1] Kulcar, B., Goricanec, D., Krope, J. (2008). Economy of exploiting heat from low-temperature geothermal sources using a heat pump. Energy and Buildings, 40 (3), 323-329. doi: 10.1016/j.enbuild.2007.02.033

[2] Randhavane, S. B., Khambete, A. K. (2017). Harnessing hydroxyl radicals generated by hydrodynamic cavitation reactor in simultaneous removal of chlorpyrifos pesticide and COD from aqueous solution. DESALINATION AND WATER TREATMENT, 82, 346-354. doi: 10.5004/dwt.2017.20965

[3] Won, S. P. (2012). Performance Analysis of an Air-Cycle Refrigeration System. Korean Journal of Air-Conditioning and Refrigeration Engineering, 24 (9), 671-678. doi: 10.6110/kjacr.2012.24.9.671

[4] Nemtinov, V. (2017). Optimization model of heat supply consumers connection schedule to the heat supply system. 17th International Multidisciplinary Scientific GeoConference SGEM2017, Informatics, Geoinformatics and Remote Sensing. doi: 10.5593/sgem2017/21/s08.128 
[5] Gaikwad, V., Ranade, V. (2016). Disinfection of Water Using Vortex Diode as Hydrodynamic Cavitation Reactor. Asian Journal of Chemistry, 28 (8), 1867-1870. doi: 10.14233/ajchem.2016.19991

[6] Amin, L. P., Gogate, P. R., Burgess, A. E., Bremner, D. H. (2010). Optimization of a hydrodynamic cavitation reactor using salicylic acid dosimetry. Chemical Engineering Journal, 156 (1), 165-169. doi: 10.1016/j.cej.2009.09.043

[7] Danfoss builds heat pump portfolio. (2006). Pump Industry Analyst, 2006 (7), 11. doi: 10.1016/ s1359-6128(06)71441-0

[8] Andreas, A. (2012). MTBE-Degradation by Hydrodynamic Induced Cavitation. Proceedings of the 8th International Symposium on Cavitation. doi: 10.3850/978-981-07-2826-7_025.

[9] Wei, J., Kawaguchi, Y., Hirano, S., Takeuchi, H. (2004). Study on a PCM Heat Storage System for Rapid Heat Supply. Heat Transfer, Volume 1, 267-274. doi: 10.1115/imece2004-61025

[10] Chen, Y., Ding, G., Shi, Y. (2009). C303 A new technology coupling with heat pump water heat, dehumidification and refrigeration (Heat Pump-1). The Proceedings of the International Conference on Power Engineering (ICOPE), 2009.3, 3-151-3-156. doi: 10.1299/jsmeicope.2009.3._3-151_

[11] Glatzmaier, G. A. (2017). Magnetic Field. Princeton University Press. doi: 10.23943/princeton/9780691141725.003.0011

[12] Pace, M. E. (2004). Liquid propane gas (lpg) storage area boiling liquid expanding vapor explosion (bleve) analysis. doi: 10.2172/820866

[13] Galeski, A., Bartczak, Z. (2003). Cavitation and cavity-free deformation of filled crystalline polymer systems. Macromolecular Symposia, 194 (1), 47-62. doi: 10.1002/masy.200390105

[14] Ghayal, D., Pandit, A. B., Rathod, V. K. (2013). Optimization of biodiesel production in a hydrodynamic cavitation reactor using used frying oil. Ultrasonics Sonochemistry, 20 (1), 322-328. doi: 10.1016/ j.ultsonch.2012.07.009

[15] Caulk, R. A., Tomac, I. (2017). Reuse of abandoned oil and gas wells for geothermal energy production. Renewable Energy, 112, 388-397. doi: 10.1016/j.renene.2017.05.042 